\title{
Review of Feature Selection Methods in Medical Image Processing
}

\author{
Adegoke, B.O ${ }^{1}$, Ola, B.O. ${ }^{2}$ and Omotayo, M.E ${ }^{3}$ \\ ${ }^{1}$ (Department of Computer Science and Engineering, Ladoke Akintola University of Technology, Nigeria) \\ ${ }^{2}$ (Department of computer Engineering, Osun State Polytechnic, Iree, Osun State, Nigeria) \\ ${ }^{3}$ (Department of Electrical Electronics, Faculty of Engineering, Osun State Polytechnic, Iree, Osun State,
} Nigeria)

\begin{abstract}
This seminar reviews features selection method of medical image processing. How image is selected by using different types of method like: screening, scanning and selecting. We discussed on feature selection technique which is widely used for data mining and knowledge discovery and it allows elimination of (irrelevant/redundant) features, whilst retaining the underlying discriminatory information, feature selection implies less data transmission and efficient data mining. It emphasized the need for further research in the field of pattern recognition that can effectively determine the situation with captured portion of human body.
\end{abstract}

Keywords: - image processing, feature selection, medical image, screening, scanning, selecting.

\section{INTRODUCTION}

Medical imaging modalities are used to probe the human body. Interpretation of the resulting images requires sophisticated image processing methods that enhance visual interpretation, and image analysis methods that provide automated or semi-automated tissue detection, measurement and characterization, multiple transformations will be needed in order to extract the data of interest from an image, and a hierarchy in the processing steps will be evident, e.g. enhancement will precede restoration, which will precede analysis [1].

\section{MEDICAL IMAGE PROCESSING}

Image processing in medical diagnosis involve stages such as image capture, image enhancement, image segmentation and feature extraction [2,3] Figure 1 shows a general description of lung cancer detection system that contains four basic stages. As depicted in fig 1, medical image processing contains different stages. The first stage starts with taking a collection of image (normal and abnormal) from the available client. The second stage applies several techniques of image enhancement, to get best level of quality and clearness. The third stage applies image segmentation algorithms which play an effective rule in image processing stages, and the fourth stage obtain the general features from enhanced segmented image which gives indicators of normality or abnormality of images.

\section{FEATURE SELECTION IN MEDICAL IMAGE PROCESSING}

Feature selection is a dimensionality reduction technique widely used for data mining and knowledge discovery and it allows elimination of (irrelevant/redundant) features, whilst retaining the underlying discriminatory information, feature selection implies less data transmission and efficient data mining. It also brings potential communication advantages in terms of packet collisions, data rate, and storage [4]. Feature selection is one of the key topics in machine learning and other related fields it can remove the irrelevant even noisy features and hence improve the quality of the data set and the performance of learning systems [5]. Expeditious growth of digital image databases motivated Content Based Image Retrieval which in turn requires efficient search schemes. Low level visual features including color, texture and shape, are automatically selected to represent images [6].

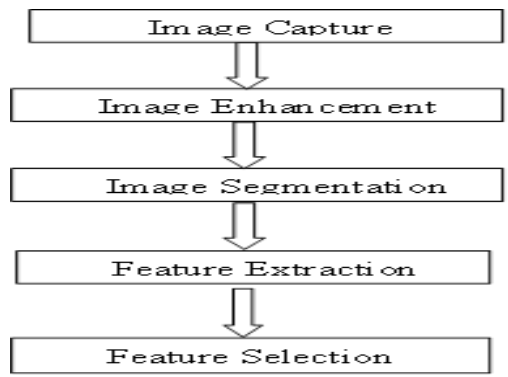

Fig. 1: Process flow in medical image processing. 


\section{Fundamental Feature Selection Methods in Medical Image Processing}

The feature selection method discussed on three steps when selecting image which are: screening, ranking and selecting. In screening, it removes unimportant and problematic predictors and records or cases, such as predictors with too many missing values or predictors with too much or too little variation to be useful. Ranking, Sorts remaining predictors and assigns ranks based on importance. Selecting: It identifies the subset of features by preserving only the most important predictors and filtering or excluding all others [7]. The Feature Selection screens, ranks, and selects are the predictors that are most important.

\section{Review Of Literature On Feature Selection Method}

Haleh and Kenneth describes part of a larger effort to apply machine learning techniques to such problems in an attempt to automatically generate and improve the classification rules required for various recognition tasks, image recognition presents a variety of difficult classification problems involving the identification of important scene components in the presence of noise, changing lighting conditions, and shifting viewpoints [8]. Since each feature used as part of a classification procedure can increase the cost and running time of a recognition system, there is strong motivation within the image processing community to design and implement systems with small feature sets. At the same time there is a potentially opposing need to include a sufficient set of features to achieve high recognition rates under difficult conditions. This has led to the development of a variety of techniques within the image processing community for finding an "optimal" subset of features from a larger set of possible features.

Sérgio et al., described the advantage of a single-valued functions that evaluate rankings to develop a family of feature selection methods based on the genetic algorithm, it improve the accuracy of content-based image retrieval systems and it also evaluate the ranking quality allows improving retrieval performance [9]. Medical images play a central role in patient diagnosis, therapy, surgical planning, medical reference, and training. With the recent boom in the availability of filmless radiology equipment, the management of digital medical mages is receiving more and more attention. Picture Archiving and Communication Systems (PACS) have been successfully introduced in many hospitals and specialized clinics, providing quick access to screening exams and integrating the actors involved in the enterprise's workflow. The radiological databases originally built for storing digital images have evolved from simple storage servers of past exams, kept for legal reasons, to active and easily accessible repositories for research and decision support.

Jaba and Shanthi, reviewed previously on continuous feature discretization and identified defining characteristics of the methods. Then propose a new supervised approach which combines discretization and feature selection to select the most relevant features which can be used for classification purpose. The classification technique to be used is Associative Classifiers [10]. Medical images are a fundamental part of medical diagnosis and treatment. These images are different from typical photographic images primarily because they reveal internal anatomy as opposed to an image of surfaces. Sasi and Kumaraswamy, said with various techniques proposed in literature for feature extraction, classification and retrieval, Content-based image retrieval (CBIR) is a widely researched area. Also discussed that Information Gain is used to achieve the structure of a feature sets to find a subset of the original feature vector for efficient computation and features are optimized using Particle Swarm Optimization (PSO) [11]. Yong Fan, et al., presented a framework for brain classification based on multi-parametric medical images, and described the method advantage of multiparametric imaging to provide a set of discriminative features for classifier construction by using a regional feature extraction method which takes into account joint correlations among different image parameters [12]. Ling-Chen et al., discussed a feature selection algorithm based on ant colony optimization (ACO), and said Image feature selection (FS) is an important task which can affect the performance of image classification and recognition [13]. Ant colony optimization (ACO) is an evolution simulation algorithm proposed by M. Dorigo et al., It has been successfully used for system fault detecting, job-shop scheduling, network load balancing, graph coloring, robotics and other combinational optimization problems. Pushpalata and Jyoti, described feature selection technique and an ensemble model proposed to improve classification accuracy. Feature selection technique is used for selecting subset of relevant features from the data set to build robust learning models and discussed further more that Classification accuracy is improved by removing most irrelevant and redundant features from the dataset and stated that Ensemble model is proposed for improving classification accuracy by combining the prediction of multiple classifiers, Three decision tree data mining classifiers were considered for classification which are CART, CHAID and QUEST [7].

Jin $\mathrm{Yu}$ et al., presented an approach that involves the analysis of Co focal Scanning Laser Tomography (CSLT) images using moment methods to derive abstract image defining features, and then the use of these features to train classifiers for automatically distinguishing CSLT images of healthy and diseased optic nerves, and investigations in feature subset selection methods for reducing the relatively large input space produced by the moment methods [14]. Vasantha et al., discussed that Breast cancer is the most common type of cancer found in women, and they proposes a image classifier to classify the mammogram images, mammogram image 
is classified into normal image, benign image and malignant image. A hybrid approach of feature selection was proposed in reduction of about $75 \%$ of the features [15].

Saravana et al., discussed about feature selection and an efficient method for feature extraction was proposed for image retrieval process and described Content-Based Image Retrieval as a technique that utilizes the visual content of an image to search for similar images in large scale image databases. Feature selection and feature extraction method were the important tasks that was considered in image retrieval process [16]. Huanzhang et al., discussed about Feature subset selection as an important subject when training classifiers in Machine Learning (ML) problems and described the fact that the complexity of the classifier parameters adjustment during training increases exponentially with the number of features. So they introduced a novel embedded feature selection method, called ESFS, which was inspired from the wrapper method SFS since it relies on the simple principle to add incrementally most relevant features [17]. Georgia et al., discussed the study of an investigated information theoretic approach to feature selection for computer-aided diagnosis, the approach was based on the mutual information (MI) concept. MI measures the general dependence of random variables without making any assumptions about the nature of their underlying relationships. They described MI that it can potentially offer some advantages over feature selection techniques that focus only on the linear relationships of variables [18]. Mohamed et al., discussed an approach which was proposed to develop a computer-aided diagnosis (CAD) system that can be very helpful for radiologist in diagnosing microcalcifications' patterns in digitized mammograms earlier and faster than typical screening programs and showed the efficiency of feature selection on the CAD system, and implemented the proposed method in four stages which are [19]:

a) The region of interest (ROI) selection of $32 \times 32$ pixels size which identifies clusters of microcalcifications,

b) The feature extraction stage based on the wavelet decomposition of locally processed image (region of interest) to compute the important features of each cluster,

c) The feature selection stage, which select the most significant features to be used in next stage, and

d) The classification stage, which classify between normal and microcalcifications' patterns and then classify between benign and malignant microcalcifications.

Guo-Zheng et al., discussed the feature selection methods using support vector machines which has obtained satisfactory results, and propose a prediction risk based on feature selection method using multiple classification support vector machines. The performance of the proposed method is compared with the previous methods of optimal brain damage based feature selection methods using binary support vector machines [4].

Shuqin et al., said feature selection techniques has been widely used in various fields and discussed a new refined feature selection module which utilizes two-step selection method in computer-aided diagnosis (CAD) system for liver disease, the method used was filter and wrapper method, Support Vector Machine (SVM) and Genetic Algorithm (GA) And stated that the advantage was to show the ability of accommodating multi feature selection search strategies and combining filter and wrapper method, especially in identifying optimal and minimal feature subsets for building the classifier [20].

Yong and Ding-gang described feature extraction and selection are of great importance in neuro image classification for identifying informative features and reducing feature dimensionality, which are generally implemented as two separate steps and presented an integrated feature extraction and selection algorithm with two iterative steps: constrained subspace learning based feature extraction and support vector machine (SVM) based feature selection [21].

Haleh and Kenneth, discussed an approach being explored to improve the usefulness of machine learning techniques for generating classification rules for complex, real world data. An approach has been implemented and tested on difficult texture classification problems. The approach involves the use of genetic algorithms as a "front end" to traditional rule induction systems in order to identify and select the best subset of features to be used by the rule induction system [8]. Feature Selection (FS) algorithms aim at choosing a reduced number of features that preserves the most relevant information of the dataset. FS is usually applied as a preprocessing step in data mining tasks by removing irrelevant or redundant features (dealing with the dimensionality curse), therefore leading to more efficient (reducing the computational cost and the amount of memory required) and accurate classification, clustering and similarity searching processes. Since each feature used as part of a classification procedure can increase the cost and running time of a recognition system, there is strong motivation within the image processing community to design and implement systems with small feature sets. At the same time there is a potentially opposing need to include a sufficient set of features to achieve high recognition rates under difficult conditions. This has led to the development of a variety of techniques within the image processing community for finding an "optimal" subset of features from a larger set of possible features. Images have a large number of features. It is important to identify and extract interesting features for a particular task in order to reduce the complexity of processing. These are attributes or portion of the image being analyzed that is most likely to give interesting rules for that problem. Not all the attributes of an image are useful for knowledge extraction. An image can be adequately represented using the attributes of its features. 
The extraction of the features from an image can be done using a variety of image processing techniques. We localize the extraction process to very small regions in order to ensure that we capture all areas.

Feature selection helps to reduce the feature space which improves the prediction accuracy and minimizes the computation time. This is achieved by removing irrelevant, redundant and noisy features .i.e., it selects the subset of features that can achieve the best performance in terms of accuracy and computation time. It performs the Dimensionality reduction. Features are generally selected by search procedures. A number of search procedures have been proposed. Popularly used feature selection algorithms are Sequential forward Selection, Sequential Backward selection, Genetic Algorithm and Particle Swarm Optimization. In this work a combined approach of Greedy stepwise method and Genetic Algorithm is proposed to select the optimal features. The selected optimal features are considered for classification.

\section{CONCLUSION}

From this study, it is discovered that selection algorithm determines the authenticity of a medical image process decisions. The performance of three different methods that is considered are: screens, ranks, and selects, which are the predictors that are most important in removing unimportant and problematic predictors and records or cases, such as predictors with too many missing values or predictors with too much or too little variation to be useful. In medical image processing, a robust and sophisticated method will be necessary such that two or three of the existing selection methods can be hybridized for better performance in real time.

\section{ACKNOWLEDGEMENT}

I want to use this avenue to appreciate the effort of Mr. Babawale, $\mathrm{K}$ for his effort to typeset this write-up.

\section{REFERENCES}

[1] Dougherty G, (2010). Image analysis in medical imaging: recent advances in selected examples. Biomedical Imaging and Intervention Journal, 1-8.

[2] Loganathan R and Kumaraswamy, (2013). Performance Evaluation of Image Compression for Medical Image. Indian Journal of Computer Science and Engineering(IJCSE), 4(2), 138-143.

[3] Hana, B., Yunjo, L. Cheryl L. G and Morris M., (2013). Age-related dedifferentiation and Compensatory Changes in the functional network underlying face processing. Neurobiology of Aging, 34(12), 27592767.

[4] Thiemjarus .S, B. P. L. Lo, Laerhoven K.V and G. Z. Yang ,(2005). Feature Selection for Wireless Sensor Networks. In Proc of $1^{\text {st }}$ International Workshop on wearable and Implatable Body Sensors Networks.

[5] Guo-Zheng Li, Jie Yang, Guo-Ping Liu and Li Xue, (2004). Feature Selection for Multi-Class Problems Using Support Vector Machines. Image Processing and Pattern Recognition, 109-111.

[6] Gnanaseker, P., Nagappan, A, Sharavanan, S, Saravanan O, Vinodkumar D., Elayabharathi T and Karthik G, (2011). Investigation on feature extraction and classification of Medical images. World Academy of Science, Engineering and Technology, 60, 327-332.

[7] Pushpalata P. and Jyoti B. G., (2012). Improving Classification Accuracy by Using Feature Selection and Ensemble Model. International Journal of Soft Computing and Engineering (IJSCE), 2(2), 380 - 386.

[8] Haleh V. and Kenneth D. J, (1992). Genetic Algorithms as a Tool for Feature Selection in Machine Learning. Artificial Intelligence, 102-109.

[9] Sérgio Francisco da Silva , Marcela Xavier Ribeiro , João do E.S. Batista Neto, Caetano Traina-Jr. ,Agma J.M. Traina, (2011). Improving the ranking quality of medical image retrieval using a genetic feature selection method. Medical Imaging, 1012-1017.

[10] Jaba S. L. and Shanthi V., (2009), International Journal of Computer Theory and Engineering, 1(2), 154158.

[11] Sasi K. M and Y.S. Kumaraswamy (2012). Medical Image Retrieval System Using PSO for Feature Selection. International Conference on Computational Techniques and Mobile Computing (ICCTMC'2012). 182-186.

[12] Yong F., Susan M., Resnick, Christos Davatzikos, (2008). Section on Biomedical Image Analysis, Department of Radiology, University of Pennsylvania, Philadelphia, PA Lab of Personality and Cognition, Intramural Research Program, National Institute on Aging, Baltimore, MD

[13] Ling C., Bolun C., and Yixin C., (2011). Image Feature Selection Based on Ant Colony Optimization.

[14] Jin Y., Syed S. R. A., and Paul H. A., (2005). A hybrid feature selection strategy for image defining features: towards interpretation of optic nerve images,

[15] Vasantha M., Dr.V.S. Bharathi, Dhamodharan R, (2010). Medical Image Feature, Extraction, Selection And Classification, International Journal of Engineering Science and Technology. 2(6), 2071-2076. 
[16] Saravana K., Sumathi A., and Latha K, (2012). Feature Selection and Extraction for Content-Based Image Retrieval, International Journal of Mathematics Trends and Technology. 3(2), 70-73.

[17] Huanzhang F, Zhongzhe X, Emmanuel D, Weibei D, and Liming C, (2009). Image Categorization Using ESFS: A New Embedded Feature Selection Method Based on SFS. 288-299.

[18] Georgia D. T., Erik D. Frederick, Mia K. Markey, and Carey E. F., (2001). Department of Biomedical Engineering, Duke University, Durham.

[19] Mohamed A. A, Wael A. M, Abo-Bakr M. Y, Yasser M. K, and Ahmed S. M, (2009). Feature Selection in Computer Aided Diagnosis System for Microcalcification Detection in Digital Mammograms. Proc of $26^{\text {th }}$ National Radio Science Conferenc (NRC2009).1-9.

[20] Shuqin W, Yan S, Qionghua W, Jun Y, Lixu G, Lijun Q and Jianrong X, (2009). Improvement of feature Selection in multi-phase CT images of hepatic lesions. Proc of the International Symposium on Bioelectronics \& Bioinformatics, 72-75.

[21] Yong F. and Dinggang S., (2009). Integrated Feature Extraction and Selection for Neuroimage classification. Proc of SPIE of Medical Imaging, 7259, 1-8. 